\title{
Mediating Effect of Executive Function on Memory in Normal Aging Adults
}

\author{
Min-Jae Kim¹, Jun Soo Kwon² and Min-Sup Shin ${ }^{2}$ \\ ${ }^{1}$ Department of Child and Adolescent Psychiatry, Seoul National University Hospital, Seoul, Republic of Korea \\ ${ }^{2}$ Department of Psychiatry, College of Medicine, Seoul National University, Seoul, Republic of Korea
}

Objective We hypothesize that the effect of aging on memory is mediated by executive function.

Methods Two hundred and thirty healthy adults (101 male, 129 female) were recruited for the study. We used a promising, newly developed, computerized neuropsychological test for the measurement of executive function and memory. The data were analyzed using structural equation modeling and path analysis.

Results The full mediation model showed a good fit to the data. However, chi-squared $\left(\chi^{2}\right)$ tests for model comparison indicated that the partial mediation model better fits our data. Thus, the partial mediation model was used as the final model. In terms of auditory-verbal memory, the effect of aging on memory was fully mediated by executive function. However, visuo-spatial memory was significantly affected both indirectly (through executive function) and directly (by aging). Gender differences were not significant in this model.

Conclusion This study demonstrated the importance of executive function in the memory functioning of normal aging adults. It is noteworthy that modality differences were found between auditory-verbal and visuo-spatial memory. Aging is not the only factor that drives memory decline, and its direct, adverse effect on memory was more prominent in the visuo-spatial memory task than auditoryverbal memory task. Since performance in both modalities is fully or partially mediated by executive function, it is important to train normal aging adults in executive control skills, such as planning, strategy formation, and rapid decision making.

Psychiatry Investig 2013;10:108-114

Key Words Memory, Executive function, Trail making test, Aging, Neuropsychological test.

\section{INTRODUCTION}

\section{Aging and memory}

As people age, they feel that everything is not what it used to be, especially in terms of memory. People often assume that their memory will decline permanently and inevitably with age. However, previous research in this area indicates that while cognitive function does tend to decline with age, such cognitive decline is associated with a variety of complex cognitive variables, not only aging. In his recent short review, Salthouse ${ }^{1}$ reported that statistically controlling for neuropsychological variables like reasoning, speed, and vocabulary

Received: October 22, 2012 Revised: December 10, 2012

Accepted: December 18, 2012 Available online: May 30, 2013

$\bowtie$ Correspondence: Min-Sup Shin, PhD

Department of Psychiatry, Seoul National University College of Medicine, 101 Daehak-ro, Jongno-gu, Seoul 110-744, Republic of Korea

Tel: +82-2-2072-2454, Fax: +82-2-747-9063, E-mail: shinms@snu.ac.kr

(c) This is an Open Access article distributed under the terms of the Creative Commons Attribution Non-Commercial License (http://creativecommons.org/licenses/by$\mathrm{nc} / 3.0$ ) which permits unrestricted non-commercial use, distribution, and reproduction in any medium, provided the original work is properly cited. substantially reduces the apparent effects of aging on various measures of memory. In addition, older people's memory performance is largely dependent on the type of memory task performed. Tasks that test implicit or procedural memory show little or no age-related decline, while episodic memory is generally much poorer in the elderly than in younger people. ${ }^{2}$ Since many controversial results regarding the relationship between aging and memory have been previously presented, it is important to consider the possible mediating or moderating factors of age-related memory decline by using various memory measures.

\section{Possible mediators}

Several different variables have been considered as potential mediators of age-related cognitive decline in previous studies; among the most promising candidates were processing speed, working memory, and executive control function. Decreases in the first factor, processing speed, are considered to be the primary driver of age-related cognitive decline. Using data from 39 different studies, Salthouse found that the 


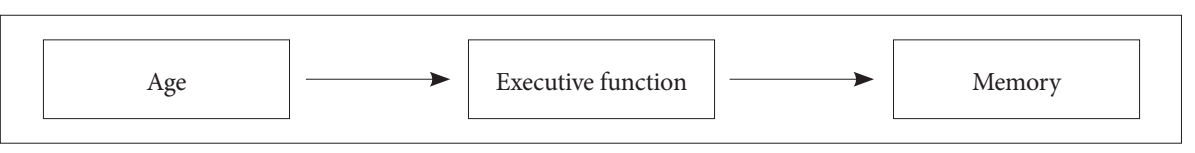

average correlation between reaction time and chronological age is $0.45 .{ }^{3}$ In later work, he suggested that decreased speed, along with age, interfered with rapid perception and decision making in complex tasks. When speed was controlled, the apparent effects of aging on cognitive function were substantially decreased. ${ }^{4}$

The second possible factor, working memory, serves as a site for temporary storage; information is actively manipulated within working memory. ${ }^{5}$ Working memory has also been found to decline with chronological age, and decreased working memory capacity affects various cognitive measures, including memory. ${ }^{6,7}$ Assessments of working memory are usually dependent on span tasks. Most previous studies have suggested that working memory span declines with age, especially for more complex tasks. However, age-related differences are minimal in simple span tasks, such as the digit span task. ${ }^{2,8}$ This indicates that working memory affects age-related memory decline through a factor other than lack of storage.

These findings indicate that executive function and executive control deserve more attention. Executive control is an effortful form of processing that affects working memory ability and performance in higher-level cognitive activities, such as problem solving. ${ }^{9}$ Executive function tends to affect all aspects of behavioral and cognitive function, albeit indirectly. ${ }^{10}$ Executive function, which encompasses processing speed and complex working memory representations that require high levels of control and manipulation, should certainly be considered as a possible comprehensive factor for the measurement of age-related decline in memory. However, it is unclear how executive function can be properly measured.

The Trail Making Test (TMT) is one instrument that could possibly be used to measure executive function. It is a very simple test, but it appears to measure various cognitive dimensions. ${ }^{11,12}$ Recent factor analysis results suggest that the TMT-A and TMT-B - among the various neuropsychological measures - can assess the constructs of speed and executive function. ${ }^{13}$ Another study demonstrated that the TMT measured shifting and sequencing. ${ }^{14}$ Those results indicated that TMT is a valuable and comprehensive measure that can test for complex executive functions, such as attention shifting, planning, and processing speed.

We hypothesized that the effect of aging on memory is mediated by executive control function and working memory, and we adopted a computerized version of the Trail Making Test as the measure of executive function.

Hypothesis: The effect of aging on memory will be mediat- ed by executive function. After controlling for the mediating effect of executive functioning, the direct effect of aging on memory decline will disappear or be substantially decreased (Figure 1).

\section{METHODS}

\section{Participants}

A general population sample of 230 healthy adults (101 men, 129 women) aged $40-59$ years (mean age $=50.44$ years, $\mathrm{SD}=5.37$ ) was recruited through online advertisements and personal contacts at companies and local sports centers. To obtain a representative study sample, participants of various educational levels were recruited from multiple cities (Seoul, GyeonGi-do, and ChungCheong-do). As the instructions for the computerized tests needed to be read, illiterate subjects were excluded, and education level was coded as 1-9 years $(\mathrm{n}=61,26.5 \%), 10-12$ years $(\mathrm{n}=75,32.6 \%)$, or $\geq 13$ years $(\mathrm{n}=94$, $40.9 \%$ ). In order to include only healthy, normally aging individuals in our sample, potential participants were screened using the Korean version of the Mini-Mental Status Examination for Dementia Screening-Short Form (SMMES-DS). ${ }^{15}$ The cutoff for dementia as measured by this instrument has been previously established as a score of $16 ;{ }^{15}$ hence, the inclusion criterion for the present study was a score of at least 16 on the SMMES-DS. In addition, subjects with any sensory deficit or a history of medical problems affecting the brain (e.g., stroke, seizure, or brain tumors) were excluded. All subjects signed an IRB-approved, written consent form (IRB approval number C-1111-084-387).

\section{Measures}

A recently developed computerized neuropsychological test battery was used. This battery of tests measured various cognitive functions, such as attention, verbal and visuo-spatial working memory, verbal and visuo-spatial memory, and executive function (Shin \& Kwon, 2013, forthcoming). To determine the reliability and concurrent validity of these measures, we recruited another sample that did not include any individuals in the present study's sample $(n=30)$ and conducted correlation analyses between these participants' scores and their scores on well-known measures of each component construct.

\section{Executive function}

The Korean version of the $\mathrm{TMT}^{16}$ was modified for the 
computerized version. In the TMT-A, integers (1-23) were shown on the computer's touchscreen, and participants were asked to touch the circled numbers in the correct numerical sequence. In the computerized version of the TMT-B, letters from the Korean alphabet (the original targets) were replaced by lyrics from the Korean national anthem. This change was made because previous research had discovered that even highly educated elderly people experienced difficulties organizing letters according to Korean alphabetical order. ${ }^{17}$ Participants touched numbers or letters according to the numberletter alternating sequence on the computer touchscreen (1동[dong]-2-해[hae]-3-물[mul]-...-13). In each trial, reaction times and errors were recorded. The correlations between the participants' scores on the new measure and those obtained on the original versions of the Korean TMT-A and TMT-B were 0.58 and 0.64 , respectively $(\mathrm{ps}<0.01, \mathrm{n}=30)$.

\section{Memory}

A well-known measure of auditory-verbal memory, the Auditory Verbal Learning Test (AVLT) ${ }^{18}$ was revised as a computerized version for use in this study. The AVLT presents lists of 15 words 5 times per trial. After a 20-minute pause, subjects are asked to recall the word lists and recognize target words among interference words. These word lists consist of random words (e.g., "coffee", "hat", or "school"). For the computerized version of this test, we created three categories of word lists - animals, transportation, and vegetables - ordered such that participants would be encouraged to use strategies to memorize them. Immediate recall was tested three times per trial, and then the delayed recall and delayed recognition trials took place 20 minutes later. The correlations between immediate recall, delayed recall, and delayed recognition scores on the one hand and the corresponding scores on the paper version of the Korean AVLT (K-AVLT) ${ }^{19}$ on the other were $0.74,0.61$, and 0.50 , respectively $(\mathrm{p} s<0.01, \mathrm{n}=30)$.

This newly developed, computerized visuo-spatial memory test contains both immediate recognition and delayed recognition trials. Participants were shown a $2 \times 3$ grid on the screen. Each square of the grid contained figures constructed out of lines, circles, triangles, and rectangles. Therefore, there were six total figures, presented for 10 seconds each. After the figures disappeared, the empty grid appeared on the screen, and the subject was asked to find the figures among the distraction targets and to replace the figures where they were seen before. Scores take into account both the recall of which six figures were on the screen (1 point per figure) and the placement of those figures into the correct squares of the grid ( 1 point per figure). The total score range is $0-12$, or $0-2$ points per figure. To test the concurrent validity, we conducted correlation analysis between scores on this scale and the well-known visual memory measure K-CFT. ${ }^{19}$ The correlation between immediate recognition (as measured by our test) and immediate recall (as measured by K-CFT) was 0.48 $(\mathrm{p}<0.01, \mathrm{n}=30)$, and the correlation between delayed recognition (as measured by our test) and delayed recall (as measured by K-CFT) was $0.48(\mathrm{p}<0.05, \mathrm{n}=30)$.

\section{Analysis}

SPSS for Windows (Version 19.0) and AMOS for Windows (Version 19.0) were used for correlation and path analysis. Structural equation modeling (SEM) was conducted to ascertain whether the effect of age on memory was mediated by executive function.

The first step in testing whether model fit is adequate is obtaining a nonsignificant chi-squared $\left(\chi^{2}\right)$ test result. However, this largely depends on sample size, so we also looked for other indices of fit. Following the guidelines of $\mathrm{Hu}$ and Bentler, ${ }^{20}$ values of the comparative fit index (CFI) and Tucker-Lewis Index (TLI) close to 0.95 and a root mean square error of approximation (RMSEA) value below 0.06 are all indicative of good model fit. We employed traditional $\chi^{2}$ comparisons between the nest and nested models to obtain a more parsimonious model. To test the differences between men and women, we conducted multi-group path analysis.

\section{RESULTS}

The correlations between the variables included in the model are shown in Table 1. All observed correlations were statistically significant at the 0.05 level.

\section{Model tests}

The $\chi^{2}$ test statistics and other fit indices of the two competing models are presented in Table 2. The first model is the hypothesized full mediation model (Figure 2). The $\chi^{2}$ test values were not significant, and all fit indices satisfied the criteria for acceptability $\left(\chi^{2}=19.60, \mathrm{df}=17, \mathrm{p}=0.295\right.$, TLI $=0.994$, CFI= 0.997 , RMSEA $=0.026$ ). Therefore, the model in which the effects of aging on memory decline are fully mediated by executive function is sufficient to explain our data.

In order to determine the direct effect of aging on memory, we created a partial mediation model, Model 2 (Figure 3). Model 1 was nested in Model 2 so that we could compare these models by using the $\chi^{2}$ test of difference. We created Model 2 by adding two additional pathways to Model 1; thus, Model 2 had two additional restrictions and fewer free parameters. Compared with Model 1, Model 2 lost two degrees of freedom, which resulted in the following values: $\Delta \chi^{2}=7.14$ $(\mathrm{p}<0.05)\left(\chi^{2}\right.$ critical value $=5.99$ at the $\mathrm{p}<0.05$ level $) . \Delta \chi^{2}$ was significant at the 0.05 level, indicating that Model 2 provided 
Table 1. Correlation matrix from Structural Equation Modeling analysis

\begin{tabular}{|c|c|c|c|c|c|c|c|c|}
\hline & 1 & 2 & 3 & 4 & 5 & 6 & 7 & 8 \\
\hline 1. AGE & 1 & & & & & & & \\
\hline 2. VM_IR & $-0.226^{* *}$ & 1 & & & & & & \\
\hline 3.VM_DR & $-0.223^{* *}$ & $0.766^{* *}$ & 1 & & & & & \\
\hline 4.VM_DRG & $-0.164^{*}$ & $0.613^{* *}$ & $0.587^{* *}$ & 1 & & & & \\
\hline 5. VSM_IRG & $-0.370^{* *}$ & $0.385^{* *}$ & $0.366^{* *}$ & $0.356^{* *}$ & 1 & & & \\
\hline 6. VSM_DRG & $-0.376^{* *}$ & $0.390^{* *}$ & $0.376^{* *}$ & $0.408^{* *}$ & $0.772^{* *}$ & 1 & & \\
\hline 7. TMT-A & $0.352^{* *}$ & $-0.339 * *$ & $-0.328^{* *}$ & $-0.299^{* *}$ & $-0.276^{* *}$ & $-0.322^{* *}$ & 1 & \\
\hline 8. TMT-B & $0.401^{* *}$ & $-0.298^{* *}$ & $-0.282^{* *}$ & $-0.251^{* *}$ & $-0.370^{* *}$ & $-0.403^{* *}$ & $0.555^{* *}$ & 1 \\
\hline
\end{tabular}

Higher scores on the TMT-A and TMT-B indicate impairment. ${ }^{*} \mathrm{p}<0.05$ (2-tailed), ${ }^{* *} \mathrm{p}<0.01$. VM: verbal memory, VSM: visuo-spatial memory, IR: Immediate recall, DR: delayed recall, IRG: immediate recognition, DRG: delayed recognition, TMT: Trail Making Test

Table 2. Fit indices for competing models

\begin{tabular}{lcccc}
\hline \multicolumn{1}{c}{ Model } & $\chi^{2}(\mathrm{df})$ & TLI & CFI & RMSEA \\
\hline Full mediation model (Model 1) & $19.60(17)$ & 0.994 & 0.997 & 0.026 \\
Partial mediation model (Model 2) & $12.46(15)$ & 1.006 & 1.000 & 0.000 \\
\hline
\end{tabular}

$\chi^{2}$ : chi-squared, df: degrees of freedom, CFI: comparative fit index, TLI: Tucker-Lewis Index, RMSEA: root mean square error of approximation

Figure 2. Full mediation model. Arrows reflect relationships between variables, and standardized regression coefficients are shown next to each path. ${ }^{* * *} p<0.001$. VM: verbal memory, VSM: visuo-spatial memory, IR: immediate recall, DR: delayed recall, IRG: immediate recognition, DRG: delayed recognition, TMT: Trail Making Test. Higher scores on the TMT-A and TMT-B indicate impairment, Ex: executive function.

a better explanation of our data than Model 1. Moreover, all of the traditional fit indices were better for Model $2\left(\chi^{2}=12.46\right.$, $\mathrm{df}=15, \mathrm{p}=0.644$, TLI=1.006, CFI $=1.000$, RMSEA $=0.000$ ) than for Model 1. Therefore, the partial mediation model was accepted as the final model for this study.

\section{Path analysis for the final model}

On the basis of the literature, this model hypothesized that aging would have a negative effect on executive function, which in turn would have a negative effect on memory. All tested indirect effects were significant. In the final model, age negatively influenced executive function $(\beta=0.51, \mathrm{p}<0.001)$, and impairment in executive function affected verbal and vi- sual memory performance $(\beta=-0.47, p<0.001$; and $\beta=-0.43$, $\mathrm{p}<0.001$, respectively). The older the participant, the slower his/her performance on the TMT-A and TMT-B was. The more time participants spent on a TMT task, the poorer their performance was on verbal and visuo-spatial memory tasks. As hypothesized, after controlling for the mediating effect of executive function, the direct effect of age on verbal memory was no longer significant $(\beta=-0.02, \mathrm{p}=0.840)$. However, the direct effect of age on visuo-spatial memory was still significant $(\beta=-0.21, p=0.009)$ (The regression weights of each structural path are presented in Table 3 ). Advanced age was negatively correlated with visuo-spatial memory performance, even after we accounted for executive function. This 


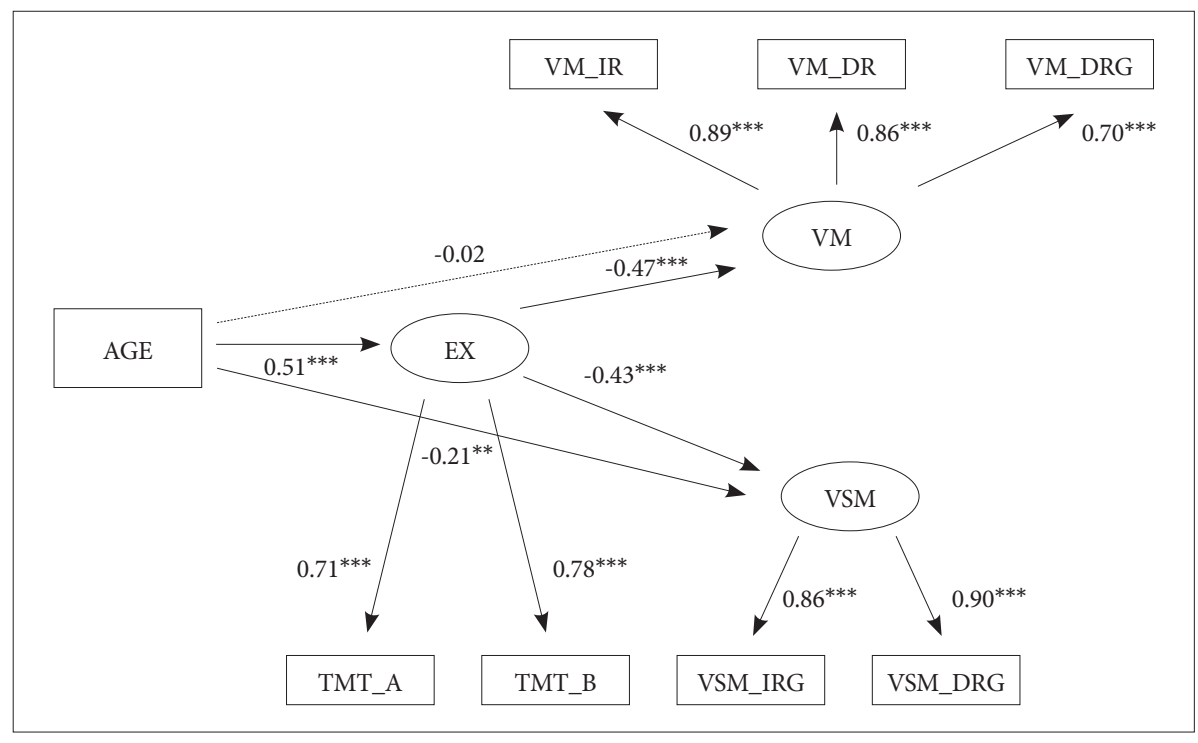

Figure 3. Partial mediation model (final model). Arrows reflect relationships between variables, and the dashed line represents a nonsignificant association. Standardized regression coefficients are shown next to each path. ${ }^{* *} \mathrm{p}<0.01,{ }^{* * *} \mathrm{p}<$ 0.001 . VM: verbal memory, VSM: visuospatial memory, IR: immediate recall, DR: delayed recall, IRG: immediate recognition, DRG: delayed recognition, TMT: Trail Making Test. Higher scores on the TMT-A and TMT-B indicate impairment, Ex: executive function.

Table 3. Estimated regression coefficients for the final model

\begin{tabular}{cllccccc}
\hline & \multirow{2}{*}{ Paths } & & $\begin{array}{c}\text { Estimated } \\
\text { regression coefficient }\end{array}$ & $\begin{array}{c}\text { Standardized } \\
\text { regression coefficient }\end{array}$ & SE & CR & p \\
\hline Age & $\rightarrow$ & EX & 2.527 & 0.506 & 0.375 & 6.733 & $* * *$ \\
EX & $\rightarrow$ & VM & -0.093 & -0.469 & 0.021 & -4.442 & $* * *$ \\
EX & $\rightarrow$ & VSM & -0.038 & -0.428 & 0.009 & -4.295 & $* * *$ \\
Age & $\rightarrow$ & VM & -0.016 & -0.017 & 0.082 & -0.202 & 0.840 \\
Age & $\rightarrow$ & VSM & -0.092 & -0.206 & 0.035 & -2.610 & 0.009 \\
\hline
\end{tabular}

Higher scores on EX indicate impairment. ${ }^{* * *} \mathrm{p}<0.001$. EX: executive function, VM: verbal memory, VSM: visuo-spatial memory

Table 4. Structural weights of the measurement model (all factor loadings constrained)

\begin{tabular}{|c|c|c|c|c|c|c|c|c|}
\hline \multirow{2}{*}{\multicolumn{3}{|c|}{ Structural path }} & \multicolumn{3}{|c|}{ Male } & \multicolumn{3}{|c|}{ Female } \\
\hline & & & \multirow{2}{*}{$\frac{\mathrm{B}}{1.583}$} & \multirow{2}{*}{$\frac{\beta}{0.373}$} & \multirow{2}{*}{$\frac{\mathrm{p}}{* * *}$} & \multirow{2}{*}{$\frac{\mathrm{B}}{2.936}$} & \multirow{2}{*}{$\frac{\beta}{0.538}$} & \multirow{2}{*}{$\frac{\mathrm{p}}{* * *}$} \\
\hline Age & $\rightarrow$ & EX & & & & & & \\
\hline EX & $\rightarrow$ & $\mathrm{VM}$ & -0.134 & -0.541 & $* * *$ & -0.076 & -0.424 & 0.003 \\
\hline EX & $\rightarrow$ & VSM & -0.055 & -0.442 & $* * *$ & -0.029 & -0.414 & 0.004 \\
\hline Age & $\rightarrow$ & $\mathrm{VM}$ & 0.022 & 0.021 & 0.849 & -0.077 & -0.078 & 0.495 \\
\hline Age & $\rightarrow$ & VSM & -0.097 & -0.183 & 0.081 & -0.084 & -0.223 & 0.053 \\
\hline
\end{tabular}

Higher scores on EX indicate impairment. ${ }^{* * *} \mathrm{p}<0.001$. B: Estimated regression coefficient, $\beta$ : Standardized regression coefficient, EX: executive function, VM: verbal memory, VSM: visuo-spatial memory

discrepancy will be explored in the Discussion.

\section{Multi-group analysis of gender differences}

To test the differences between men and women, we conducted multi-group path analysis, which tests the invariance of a structural model across subgroups. In this analysis, structural paths are compared between subgroups, and if there are no differences, cross-validation of hypothesized models between subgroups is established. ${ }^{21}$ The prerequisite before the comparison between structural paths is measurement invariance between men and women. To test the equivalence of mea- surement across groups, we imposed equality constraints on each factor loading and conducted a model test. The results showed nonsignificant $\chi^{2}$ test values, and all fit indices satisfied the criteria for goodness of fit $\left(\chi^{2}=33.202, \mathrm{df}=34, \mathrm{p}=0.507\right.$, TLI $=1.002, \mathrm{CFI}=1.000, \mathrm{RMSEA}=0.000$ ). The regression weights of each structural path are presented in Table 4. Since measurement invariance was satisfied, we imposed equality constraints on each structural path and all structural paths between men and women, and conducted a $\chi^{2}$ difference test. As described in Table 5, there were no differences in structural paths between men and women, and when we constrain all the 
Table 5. Fit indices from invariance analysis

\begin{tabular}{lcccc}
\hline $\begin{array}{c}\text { Constrained } \\
\text { structural path }\end{array}$ & $\Delta \mathrm{df}$ & $\Delta \chi^{2}$ & $\mathrm{p}$ & $\Delta \mathrm{TLI}$ \\
\hline Age $\rightarrow \mathrm{EX}$ & 1 & 0.368 & 0.544 & -0.001 \\
$\mathrm{EX} \rightarrow \mathrm{VM}$ & 1 & 1.850 & 0.174 & 0.006 \\
$\mathrm{EX} \rightarrow \mathrm{VSM}$ & 1 & 2.169 & 0.141 & -0.002 \\
Age $\rightarrow$ VM & 1 & 3.739 & 0.053 & 0.002 \\
Age $\rightarrow$ VSM & 1 & 0.036 & 0.849 & 0.003 \\
All constrained & 5 & 7.613 & 0.179 & 0.005 \\
\hline
\end{tabular}

Higher scores on EX indicate impairment. EX: executive function, VM: verbal memory, VSM: visuo-spatial memory, $\chi^{2}$ : chi-squared, df: degrees of freedom, TLI: Tucker-Lewis Index

paths in the models, the overall goodness of model fit was not changed significantly $\left(\Delta \chi^{2}=7.613, p=0.179, \Delta T L I=0.005\right)$. In sum, the hypothesized model was cross-validated among men and women, and the research model can be applied to both middle-aged men and women.

\section{DISCUSSION}

The purpose of this study was to determine the relationship between normal aging and memory and to ascertain the mediating role of executive function in the memory functioning. The key factors in cognitive decline in normal aging are still unknown; the identification of these factors constitutes an area of much ongoing research. This study analyzed 230 healthy, normal aging adults. The study's findings indicated that aging does not have a direct effect on decline in memory but that it influences memory indirectly through executive function, especially in terms of verbal memory. Moreover, this structural model was cross-validated for men and women. It is noteworthy that there are modality differences in the influence of aging on memory. In the case of auditory-verbal memory tasks, aging was fully mediated by executive function, and after we controlled for executive function, the effect of aging on memory was not significant. However, in visuo-spatial memory tasks, age still had an influence on memory even after controlling for executive function.

Studies on hemispheric asymmetry and aging have indicated that the right hemisphere is affected more by aging than the left; the former hemisphere is also known as the main locus of pictorial and spatial information processing. ${ }^{22}$ In this study, according to the Wechsler Adult Intelligence Scale, aging participants were less impaired in terms of Verbal than Performance IQ. Recent comparative studies on the effect of aging on working memory have shown differences between the verbal and visuo-spatial domains. These studies have also shown that age influences the visuo-spatial domain more strongly than the verbal domain in terms of both accuracy and response time. ${ }^{23}$ Previous studies on aging and verbal ability suggest that semantic and lexical knowledge accumulates during adulthood and remains stable until about 90 years of age. ${ }^{24}$ It seems that verbal memory and verbal ability are not significantly affected by aging. Therefore, if people find it more difficult to memorize verbal material as they age, the cause is likely problems with executive function if other memory-related disorders are absent.

The limitations of this study included the lack of specific information of individual factors like intellectual functioning, verbal ability, and processing speed. A previous study showed that measures of executive function, including the TMT, can be largely explained by individual cognitive variables like fluid general intelligence, perceptual speed, and vocabulary. ${ }^{25}$ It is possible that executive function represents general intelligence and that cognitive function itself might affect age-related memory decline. Therefore, in future research, various moderating and mediating variables should be considered and tested separately. In addition, since the TMT (which was employed to measure executive function) also requires visuospatial skills, the use of exclusively verbal and visuo-spatial measures would be helpful for the clarification of betweenmodality differences.

The results of this study suggest that aging is not the core factor driving memory decline and that executive function is a more important factor. Therefore, if sufficient time is given for strategy formation and memorization, older people may do as well as younger people, at least on verbal tasks. This finding indicates the importance of developing various cognitive training programs especially focused on processing speed, planning, and strategy. Though we conducted reliability and validity testing, since we used newly developed, computerized measures, these tests should be replicated by future research.

\section{Acknowledgments}

This study was supported by a grant from Brain Medic for an incorporated $R \& D$ project.

\section{REFERENCES}

1. Salthouse TA. Selective review of cognitive aging. J Int Neuropsychol Soc 2010;16:754-760.

2. Zacks RT, Hasher L, Li KZH. Human Memory. In: Craik FIM, Salthouse TA, editors. The Handbook of Aging and Cognition. Mahwah: Lawrence Erlbaum Associates, 2000, p.293-357.

3. Salthouse TA. Speed of Behavior and its Implications for Cognition. In: Schaie JEBKW, editor. Handbook of the Psychology of Aging, 2nd Edition. New York: Van Nostrand Reinhold Co, 1985, p.400-426.

4. Salthouse TA. The processing-speed theory of adult age differences in cognition. Psychol Rev 1996;103:403-428.

5. Baddeley AD, Hitch G. Working Memory. In: Bower GH, editor. The Psychology of Learning and Motivation. London: Academic Press, 1974, p.47-89.

6. Lewis KL, Zelinski EM. List and text recall differ in their predictors: 
replication over samples and time. J Gerontol B Psychol Sci Soc Sci 2010; 65:449-458.

7. Borella E, Carretti B, De Beni R. Working memory and inhibition across the adult life-span. Acta Psychol (Amst) 2008;128:33-44.

8. Bopp KL, Verhaeghen P. Aging and verbal memory span: a meta-analysis. J Gerontol B Psychol Sci Soc Sci 2005;60:223-233.

9. Craik FI. Memory changes in normal and pathological aging. Can J Psychiatry 2008;53:343-345.

10. Lezak MD, Howieson DB, Loring DW. Neuropsychological Assessment. New York: Oxford University Press; 2004.

11. Misdraji EL, Gass CS. The trail making test and its neurobehavioral components. J Clin Exp Neuropsychol 2010;32:159-163.

12. Salthouse TA. What cognitive abilities are involved in trail-making performance? Intelligence 2011;39:222-232.

13. Dowling NM, Hermann B, La Rue A, Sager MA. Latent structure and factorial invariance of a neuropsychological test battery for the study of preclinical Alzheimer's disease. Neuropsychology 2010;24:742-756.

14. Atkinson TM, Ryan JP, Lent A, Wallis A, Schachter H, Coder R. Three trail making tests for use in neuropsychological assessments with brief intertest intervals. J Clin Exp Neuropsychol 2010;32:151-158.

15. Kim TH, Jhoo JH, Park JH, Kim JL, Ryu SH, Moon SW, et al. Korean version of mini mental status examination for dementia screening and its' short form. Psychiatry Investig 2010;7:102-108.

16. Lee JH, Lee KU, Lee DY, Kim KW, Jhoo JH, Kim JH, et al. Development of the Korean version of the Consortium to Establish a Registry for Alzheimer's Disease Assessment Packet (CERAD-K): clinical and neuropsychological assessment batteries. J Gerontol B Psychol Sci Soc
Sci 2002;57:47-53

17. Kim HJ, Baek MJ, Chang Y, Jang IM, Ryu HJ, Kim M, et al. Comparison between the original version of trail making test with two Koreantrail making tests. Dement Neurocognitive Disord 2011;10:95-101.

18. Rey A. L'examen clinique en psychologie. Paris: Presses Universitaire de France; 1964.

19. Kim HK. Assessment of memory disorders using Rey-Kim memory test. J Rehab Psychol 2001;8:29-48.

20. Hu LT, Bentler PM. Cutoff criteria for fit indexes in covariance structure analysis: Conventional criteria versus new alternatives. Struct Equ Modeling 1999;6:1-55.

21. Byrne BM. Structural Equation Modeling with AMOS - Basic Concepts, Applications, and Programming. Mahwah: Lawrence Erlbaum Associates; 2001.

22. Goldstein G, Shelly C. Does the right hemisphere age more rapidly than the left? J Clin Neuropsychol 1981;3:65-78.

23. Bopp KL, Verhaeghen P. Age-related differences in control processes in verbal and visuospatial working memory: Storage, transformation, supervision, and coordination. J Gerontol B Psychol Sci Soc Sci 2007; 62:P239-P246.

24. Burke DM, Shafto MA. Language and Aging. In: Craik FIM, Salthouse TA, editors. The Handbook of Aging and Cognition. Mahwah: Lawrence Erlbaum Associates, 2000, p.373-443.

25. Salthouse TA, Atkinson TM, Berish DE. Executive functioning as a potential mediator of age-related cognitive decline in normal adults. J Exp Psychol Gen 2003;132:566-594. 